\title{
Sensitivity Analysis, a Powerful System Validation Technique
}

\author{
Eric D. Smith, Ferenc Szidarovszky, William J. Karnavas and A. Terry Bahill"
}

Systems and Industrial Engineering, University of Arizona, Tucson, AZ 85721-0020, USA

\begin{abstract}
A sensitivity analysis is a powerful technique for understanding systems. This heuristic paper shows how to overcome some of the difficulties of performing sensitivity analyses. It draws examples from a broad range of fields: bioengineering, process control, decision making and system design. In particular, it examines sensitivity analyses of tradeoff studies. This paper generalizes the important points that can be extracted from the literature covering diverse fields and long time spans. Sensitivity analyses are particularly helpful for modeling systems with uncertainty.
\end{abstract}

Keywords: Modeling, sensitivity, tradeoff studies, trade studies, verification, validation.

\section{INTRODUCTION}

You should perform a sensitivity analysis anytime you create a model, write a set of requirements, design a system, make a decision, do a tradeoff study, originate a risk analysis or want to discover cost drivers. In a sensitivity analysis, you change values of parameters or inputs (or architectural features) and measure changes in outputs or performance indices. The results of a sensitivity analysis can be used to validate a model, warn of unrealistic model behavior, point out important assumptions, help formulate model structure, simplify a model, suggest new experiments, guide future data collection efforts, suggest accuracy for calculating parameters, adjust numerical values of parameters, choose an operating point, allocate resources, detect critical criteria, suggest tolerance for manufacturing parts and identify cost drivers.

A sensitivity analysis tells which parameters are the most important and most likely to affect system behavior and/or predictions of the model. Following a sensitivity analysis, values of critical parameters can be refined while parameters that have little effect can be simplified or ignored. In the manufacturing environment, they can be used to allocate resources to critical parts allowing casual treatment of less sensitive parts. If the sensitivity coefficients are calculated as functions of time, it can be seen when each parameter has the greatest effect on the output function of interest. This can be used to adjust numerical values for the parameters. The values of the parameters should be chosen to match the physical data at the times when they have the most effect on the output.

The earliest sensitivity analyses that we have found are the genetics studies on the pea reported by Gregor Mendel in 1865 [1] and the statistics studies on the Irish hops crops by Gosset writing under the pseudonym Student around 1890 [2]. Since then sensitivity analyses have been used to analyze networks and feedback amplifiers [3], to validate social

*Address correspondence to this author at the Systems and Industrial Engineering, University of Arizona, Tucson, AZ 85721-0020, USA; Tel: (520) 621-6561; Fax: (520) 621-6555; E-mail: terry@sie.arizona.edu models [4], engineering models [5] and physiological models [6-9], to target disease treatment [10], in numerical computations [11], expert systems [12-14], discrete event simulations [15], where the techniques are called response surface methodology [16, 17], frequency domain experiments [18-20], and perturbation analysis $[21,22]$. When changes in the parameters cause discontinuous changes in system properties, the sensitivity analysis is called that of singular perturbations [23]. Sensitivity functions are used in adaptive control systems $[24,25]$. In linear programming problems sensitivities are referred to as marginal values or the shadow price of each constraint and are interpreted as the increase in the optimal solution obtainable by each additional unit added to the constraint [26].

Statistical techniques can be used when the model is so complex that computing the performance indices is very difficult. For such complex models, the inputs are described with probability distributions and then the induced uncertainly in the performance indices is analyzed: this approach is known as probabilistic sensitivity analysis [27].

There is a large literature on global sensitivity analysis and regression/correlation techniques for large computer simulation models [17]. We will not deal with such complex simulations in this paper. The purpose of this paper is to present simple techniques that will increase intuition and understanding of systems.

There are many common ways to do sensitivity analyses. A partial derivative can be a sensitivity function for a system described by equations. Otherwise, spreadsheets, like Excel, are convenient for doing sensitivity analyses of systems that are not described by equations. This paper explores many techniques for doing sensitivity analyses.

There are two classes of sensitivity functions: analytic and empirical. Analytic sensitivity functions are used when the system under study is relatively simple, well defined, and mathematically well behaved (e.g. continuous derivatives). These generally take the form of partial derivatives. They are convenient, because once derived they can often be used in a broad range of similar systems and are easily adjusted for changes in all model parameters. They also have an advan- 
tage in that the sensitivity of a system to a given parameter is given as a function of all other parameters, including time or frequency (depending on the model of the system), and can be plotted as functions of these variables.

Empirical sensitivity functions are often just point evaluations of a system's sensitivity to a given parameter(s) when other parameters are at known, fixed values. Empirical sensitivity functions can be estimated from the point sensitivity evaluations over a range of the values of the parameters. They are generally determined by observing the changes in output of a computer simulation as model parameters are varied from run to run. Their advantage is that they are often simpler (or more feasible) than their analytic counterparts or can even be determined for an unmodeled physical system. If the physical system is all that is available, the system output is monitored as the inputs and parameters are changed from their normal values.

\section{ANALYTIC SENSITIVITY FUNCTIONS}

This section will explain three different analytic sensitivity functions. They are all based on finding the partial derivative of a mathematical system model with respect to some parameter. Short examples are then given for each particular form of a sensitivity function.

\subsection{The Absolute-Sensitivity Function}

The absolute sensitivity of the function $F$ to variations in the parameter $\alpha$ is given by

$S_{\alpha}^{F}=\left.\frac{\partial F}{\partial \alpha}\right|_{\mathrm{NOP}}$

where NOP means the partial derivative is evaluated at the Normal Operating Point (NOP) where all the parameters have their normal values. Of course, the function $F$ must be differentiable with respect to $\alpha$. In this paper, the function $F$ may also be a function of other parameters such as time, frequency or temperature. Absolute-sensitivity functions are useful for calculating output errors due to parameter variations and for assessing the times at which a parameter has its greatest or least effect. Absolute sensitivities are also used in adaptive control systems.

The following two examples show the use of absolutesensitivity functions. The first shows how to tailor the output of a process and the second shows how to see when a parameter has its greatest effect.

\section{Example 1. A Process Model}

Assume that the function

$$
f(x, y)=z=A x^{2}+B y^{2}+C x y+D x+E y+F
$$

models a process, where $x$ and $y$ are the inputs (raw material) and $A$ to $F$ are models of the system parameters. Let the normal operating point be

$\left(x_{0}, y_{0}\right)=(1,1), A_{0}=1, B_{0}=2, C_{0}=3, D_{0}=-5, E_{0}=-7, F_{0}=8$, producing $z_{0}=2$.
Suppose that the output $z$ is a valuable commodity (perhaps a potion). What is the easiest way (the smallest change in one of these eight operating parameters) to increase the quantity of $z$ that is being produced? This sounds like a natural problem for absolute-sensitivity functions.

$$
\begin{aligned}
& S_{A}^{z}=\left.\frac{\partial z}{\partial A}\right|_{\mathrm{NOP}}=x_{0}^{2}=1, \\
& S_{B}^{z}=\left.\frac{\partial z}{\partial B}\right|_{\mathrm{NOP}}=y_{0}^{2}=1, \\
& S_{C}^{z}=\left.\frac{\partial z}{\partial C}\right|_{\mathrm{NOP}}=x_{0} y_{0}=1, \\
& S_{D}^{z}=\left.\frac{\partial z}{\partial D}\right|_{\mathrm{NOP}}=x_{0}=1, \\
& S_{E}^{z}=\left.\frac{\partial z}{\partial E}\right|_{\mathrm{NOP}}=y_{0}=1, \\
& S_{F}^{z}=\left.\frac{\partial z}{\partial F}\right|_{\mathrm{NOP}}=1, \\
& S_{x}^{z}=\left.\frac{\partial z}{\partial x}\right|_{\mathrm{NOP}}=2 A_{0} x_{0}+C_{0} y_{0}+D_{0}=0, \\
& S_{y}^{z}=\left.\frac{\partial z}{\partial y}\right|_{\mathrm{NOP}}=2 B_{0} y_{0}+C_{0} x_{0}+E_{0}=0 .
\end{aligned}
$$

Evaluated at $\left(x_{0}, y_{0}\right)=(1,1)$, the six coefficients are equally sensitive: increase any coefficient by one unit and the output increases by one unit. Since the derivatives with respect to $x$ and $y$ are equal to zero, to analyze sensitivity with respect to these variables we need to use higher order partial derivatives, as will be shown later.

What about interactions? Could we do better by changing two parameters at the same time? Interaction terms are characterized by, for example, the partial derivative of $\mathrm{z}$ with respect to $\mathrm{x}$ containing $y$. The interactions can be bigger than the first-order partial derivatives. You can see that the above partial derivatives with respect to the coefficients contain the operating point coordinates and the partial derivatives with respect to the operating point coordinates depend on the coefficients. Therefore, we should expect the interactions to be significant. Of the 64 possible second-partial derivatives, only the following are nonzero.

$$
\begin{aligned}
& S_{x-A}^{z}=\left.\frac{\partial^{2} z}{\partial x \partial A}\right|_{\mathrm{NOP}}=2 x_{0}=2, \\
& S_{x-C}^{z}=\left.\frac{\partial^{2} z}{\partial x \partial C}\right|_{\mathrm{NOP}}=y_{0}=1, \\
& S_{x-D}^{z}=\left.\frac{\partial^{2} z}{\partial x \partial D}\right|_{\mathrm{NOP}}=1, \\
& S_{y-B}^{z}=\left.\frac{\partial^{2} z}{\partial y \partial B}\right|_{\mathrm{NOP}}=2 y_{0}=2, \\
& S_{y-C}^{z}=\left.\frac{\partial^{2} z}{\partial y \partial C}\right|_{\mathrm{NOP}}=x_{0}=1,
\end{aligned}
$$


$S_{y-E}^{z}=\left.\frac{\partial^{2} z}{\partial y \partial E}\right|_{\mathrm{NOP}}=1$

$S_{x-y}^{z}=\left.\frac{\partial^{2} z}{\partial x \partial y}\right|_{\mathrm{NOP}}=C_{0}=3$,

$S_{x^{2}}^{z}=\left.\frac{\partial^{2} z}{\partial x^{2}}\right|_{\mathrm{NOP}}=2 A_{0}=2$,

$S_{y^{2}}^{z}=\left.\frac{\partial^{2} z}{\partial y^{2}}\right|_{\mathrm{NOP}}=2 B_{0}=4$

By the theory of Young, we know that if the partial derivatives are continuous, then $\frac{\partial z^{2}}{\partial x \partial A}=\frac{\partial z^{2}}{\partial A \partial x}$ etc. so we do not have to calculate any more partial derivatives. Five of these mixed second-partial derivatives are bigger than the first-partial derivatives.

At the normal operating point, $z_{0}=2$. If we increase $A$ by one unit, then $A=2$, this increases $z=3$, and $\Delta z=1$. If we increase $x$ by one unit (this is too large of a step for a sensitivity analysis, but the math is convenient), then $x=2, z$ $=3$, and again $\Delta z=1$. However, if we simultaneously increase both $x$ and $A$ by one unit each, $A=2, x=2, z=7$, and $\Delta z=5$. The interaction effect is big. This and two other interaction effects are shown in Table $\mathbf{1}$.
Here we have treated the second partial derivative $\frac{\partial^{2} z}{\partial y^{2}}$ as an interaction term. Karnavas, Sanchez and Bahill [14], explain how this could be the result of a quadratic nonlinearity.

How about the third-order partial derivatives? e.g. $\frac{\partial^{3} z}{\partial x^{2} \partial A}=2$. Let $x=3$ and $A=2, z=15$ and $\Delta z=13$. This is a bigger output boost than that produced by the sum of changes in any three parameters. Three of the third-order partial derivatives are greater than zero. One is equal to 1 , and two are equal to 2 . All of the fourth-order partial derivatives are zero.

\section{Example 2. A Double-Pole System with a Time-Delay}

Next, we will show an example of using an absolutesensitivity function to determine when a parameter has its greatest effect. For this example, we will use Stark's [28] transfer function for the crayfish escape behavior, namely

$$
M(s)=\frac{Y(s)}{R(s)}=\frac{K e^{-\theta s}}{(\tau s+1)^{2}}
$$

and find when the parameter $K$ has its greatest effect on the step response of the system. If we let the input be a unit step,

Table 1. Effects of Individual and Combined Parameter Changes

\begin{tabular}{|c|c|c|c|c|c|}
\hline Partial Derivatives & Normal Values & Values Increased by One Unit & New $z$ Values & $\Delta z$ & Total Change in $z$ \\
\hline$\frac{\partial z}{\partial A}$ & $A=1$ & $A=2$ & 3 & 1 & \multirow{2}{*}{$\sum \Delta z=2$} \\
\hline$\frac{\partial z}{\partial x}$ & $x=1$ & $x=2$ & 3 & 1 & \\
\hline$\frac{\partial^{2} z}{\partial x \partial A}$ & $\begin{array}{l}A=1 \\
x=1\end{array}$ & $\begin{array}{l}A=2 \\
x=2\end{array}$ & 7 & 5 & 5 \\
\hline$\frac{\partial z}{\partial x}$ & $x=1$ & $x=2$ & 3 & 1 & \multirow{2}{*}{$\sum \Delta z=3$} \\
\hline$\frac{\partial z}{\partial y}$ & $y=1$ & $\mathrm{y}=2$ & 4 & 2 & \\
\hline$\frac{\partial^{2} z}{\partial x \partial y}$ & $\begin{array}{l}x=1 \\
y=1\end{array}$ & $\begin{array}{l}x=2 \\
y=2\end{array}$ & 8 & 6 & 6 \\
\hline$\frac{\partial z}{\partial y}$ & $y=1$ & $y=2$ & 4 & 2 & \multirow{2}{*}{$\sum \Delta z=4$} \\
\hline$\frac{\partial z}{\partial y}$ & $y=1$ & $y=2$ & 4 & 2 & \\
\hline$\frac{\partial^{2} z}{\partial y^{2}}$ & $y=1$ & $y=3$ & 10 & 8 & 8 \\
\hline
\end{tabular}


Table 2. Effects of Individual and Combined Parameter Changes

\begin{tabular}{|c|c|c|c|c|}
\hline Partial Derivatives & Normal Values & Values Increased by One Unit & New $z$ Values & $\Delta z$ \\
\hline \hline$\frac{\partial z}{\partial A}$ & $A=1$ & $A=2$ & 3 & 1 \\
\hline$\frac{\partial z}{\partial x}$ & $x=1$ & $x=2$ & 3 & 1 \\
\hline$\frac{\partial z}{\partial x}$ & $x=1$ & $x=2$ & 3 & 1 \\
\hline$\frac{\partial^{3} z}{\partial x^{2} \partial A}=2$ & $A=1$ & $A=2$ & 15 & 13 \\
\hline
\end{tabular}

$R(s)=\frac{1}{s}$, then the output step-response of the system is

$Y_{s r}(s)=\frac{K e^{-\theta s}}{s(\tau s+1)^{2}}$

and the absolute-sensitivity function of the step response with respect to $K$ is

$S_{K}^{Y_{s r}}(s)=\frac{e^{-\theta_{0} s}}{s\left(\tau_{0} s+1\right)^{2}}$

which transforms into

$S_{K}^{y_{s r}}(t)=1-e^{-\left(t-\theta_{0}\right) / \tau_{0}}-\frac{t-\theta_{0}}{\tau_{0}} e^{-\left(t-\theta_{0}\right) / \tau_{0}}$ for $t>\theta_{0}$

In this example, the order of taking the derivative and making the transformation can be interchanged. This function obtains its maximum value when $t$ goes to infinity. This tells us that the parameter $K$ has its greatest effect when the response reaches steady state, which is what our intuition also tells us.

\subsection{The Relative-Sensitivity Function}

If we want to compare the effects of different parameters, we should use relative-sensitivity functions. The relativesensitivity of the function $F$ to the parameter $\alpha$ evaluated at the normal operating point is given by

$\bar{S}_{\alpha}^{F}=\left.\frac{\partial F}{\partial \alpha}\right|_{\mathrm{NOP}} \frac{\alpha_{0}}{F_{0}} \approx \frac{\% \text { change in } F}{\% \text { change in } \alpha}=\frac{\frac{\Delta F}{F}}{\frac{\Delta \alpha}{\alpha}}$

where NOP and the subscripts 0 mean that all functions and parameters assume their normal operating point values [3]. Relative-sensitivity functions are formed by multiplying the partial derivative (the absolute-sensitivity function) by the normal value of the parameter and dividing by the normal value of the function. For statistical models, a relativesensitivity function can be formed by multiplying the partial derivative by the ratio of the variances [29]. Relativesensitivity functions are ideal for comparing parameters, because they are dimensionless, normalized functions. In the field of economics, the lack of dimensions of the relativesensitivity function is exploited to allow easy comparison of parameters' changes on model outputs even though the parameters may describe widely varying aspects of the model and have different units. Economists refer to the relativesensitivity function $\bar{S}_{B}^{A}$ as the elasticity of $B$ with respect to $A$, and denote it as $E_{B, A}[30]$.

\section{Example 1. The Process Model (Continued)}

Assume that the function

$$
f(x, y)=z=A x^{2}+B y^{2}+C x y+D x+E y+F
$$

models the output of a process, where the normal operating point is

$\left(x_{0}, y_{0}\right)=(1,1), A_{0}=1, B_{0}=2, C_{0}=3, D_{0}=-5, E_{0}=-7, F_{0}=8$, producing $z_{0}=2$.

What is the easiest way (smallest percent change in an operating point parameter) to increase the quantity of $z$ that is being produced? Now this problem is appropriate for relative-sensitivity functions.

$$
\begin{aligned}
& \bar{S}_{A}^{z}=\left.\frac{\partial z}{\partial A}\right|_{\mathrm{NOP}} \frac{A_{0}}{z_{0}}=x_{0}^{2} \frac{A_{0}}{z_{0}}=0.5, \\
& \bar{S}_{B}^{z}=\left.\frac{\partial z}{\partial B}\right|_{\mathrm{NOP}} \frac{B_{0}}{z_{0}}=y_{0}^{2} \frac{B_{0}}{z_{0}}=1, \\
& \bar{S}_{C}^{z}=\left.\frac{\partial z}{\partial C}\right|_{\mathrm{NOP}} \frac{C_{0}}{z_{0}}=x_{0} y_{0} \frac{C_{0}}{z_{0}}=1.5, \\
& \bar{S}_{D}^{z}=\left.\frac{\partial z}{\partial D}\right|_{\mathrm{NOP}} \frac{D_{0}}{z_{0}}=x_{0} \frac{D_{0}}{z_{0}}=-2.5, \\
& \bar{S}_{E}^{z}=\left.\frac{\partial z}{\partial E}\right|_{\mathrm{NOP}} \frac{E_{0}}{z_{0}}=y_{0} \frac{E_{0}}{z_{0}}=-3.5, \\
& \bar{S}_{F}^{z}=\left.\frac{\partial z}{\partial F}\right|_{\mathrm{NOP}} \frac{F_{0}}{z_{0}}=\frac{F_{0}}{z_{0}}=4,
\end{aligned}
$$


$\bar{S}_{x}^{z}=\left.\frac{\partial z}{\partial x}\right|_{\mathrm{NOP}} \frac{x_{0}}{z_{0}}=\left(2 A_{0} x_{0}+C_{0} y_{0}+D_{0}\right) \frac{x_{0}}{z_{0}}=0$,
$\bar{S}_{y}^{z}=\left.\frac{\partial z}{\partial y}\right|_{\mathrm{NOP}} \frac{y_{0}}{z_{0}}=\left(2 B_{0} y_{0}+C_{0} x_{0}+E_{0}\right) \frac{y_{0}}{z_{0}}=0$.

Evaluated at $\left(x_{0}, y_{0}\right)=(1,1), \quad \bar{S}_{F}^{z}=\left.\frac{\partial z}{\partial F}\right|_{\mathrm{NOP}} \frac{F_{0}}{z_{0}}=\frac{F_{0}}{z_{0}}=4$, is the largest. Therefore, we should increase $F$ if we wish to increase $z$.

What about interactions? Could we do better by changing two parameters at the same time? Only the following second-order relative-sensitivity functions are non-zero.

$$
\begin{aligned}
& \bar{S}_{x-A}^{z}=\left.\frac{\partial^{2} z}{\partial x \partial A}\right|_{\mathrm{NOP}} \quad \frac{x_{0} A_{0}}{z_{0}^{2}}=\frac{2 x_{0}^{2} A_{0}}{z_{0}^{2}}=0.5, \\
& \bar{S}_{x-C}^{z}=\left.\frac{\partial^{2} z}{\partial x \partial C}\right|_{\mathrm{NOP}} \frac{x_{0} C_{0}}{z_{0}^{2}}=\frac{x_{0} y_{0} C_{0}}{z_{0}^{2}}=0.75, \\
& \bar{S}_{x-D}^{z}=\left.\frac{\partial^{2} z}{\partial x \partial D}\right|_{\mathrm{NOP}} \frac{x_{0} D_{0}}{z_{0}^{2}}=\frac{x_{0} D_{0}}{z_{0}^{2}}=-1.25, \\
& \bar{S}_{y-B}^{z}=\left.\frac{\partial^{2} z}{\partial y \partial B}\right|_{\mathrm{NOP}} \frac{y_{0} B_{0}}{z_{0}^{2}}=\frac{2 y_{0}^{2} B_{0}}{z_{0}^{2}}=1, \\
& \bar{S}_{y-C}^{z}=\left.\frac{\partial^{2} z}{\partial y \partial C}\right|_{\mathrm{NOP}} \frac{y_{0} C_{0}}{z_{0}^{2}}=\frac{x_{0} y_{0} C_{0}}{z_{0}^{2}}=0.75,
\end{aligned}
$$

$$
\begin{aligned}
& \bar{S}_{y-E}^{z}=\left.\frac{\partial^{2} z}{\partial y \partial E}\right|_{\mathrm{NOP}} \frac{y_{0} E_{0}}{z_{0}^{2}}=\frac{y_{0} E_{0}}{z_{0}^{2}}=-1.75, \\
& \bar{S}_{x-y}^{z}=\left.\frac{\partial^{2} z}{\partial x \partial y}\right|_{\mathrm{NOP}} \frac{x_{0} y_{0}}{z_{0}^{2}}=\frac{x_{0} y_{0} C_{0}}{z_{0}^{2}}=0.75, \\
& \bar{S}_{x^{2}}^{z}=\left.\frac{\partial^{2} z}{\partial x^{2}}\right|_{\mathrm{NOP}} \frac{x_{0}^{2}}{z_{0}^{2}}=\frac{2 x_{0}^{2} A_{0}}{z_{0}^{2}}=0.5, \\
& \bar{S}_{y^{2}}^{z}=\left.\frac{\partial^{2} z}{\partial y^{2}}\right|_{\mathrm{NOP}} \frac{y_{0}^{2}}{z_{0}^{2}}=\frac{2 y_{0}^{2} B_{0}}{z_{0}^{2}}=1 .
\end{aligned}
$$

None of these is bigger than $\bar{S}_{F}^{z}=\left.\frac{\partial z}{\partial F}\right|_{\text {NOP }} \frac{F_{0}}{z_{0}}=\frac{F_{0}}{z_{0}}=4$.

In summary, using absolute-sensitivity functions, the second-order and third-order terms were the most important, but using relative-sensitivity functions, the coefficient $F$ was the most important. The absolute and relative-sensitivity functions give different results. The absolute-sensitivity functions show which parameters have the greatest affect on the output for a fixed size change in the parameters, whereas the relative-sensitivity functions show which parameters have the greatest affect on the output for a certain percent change in the parameters.

\section{Example 2. A Double-Pole System with a Time-Delay (Continued)}

Let us now show how to use relative-sensitivity functions to compare parameters. Consider the transfer function

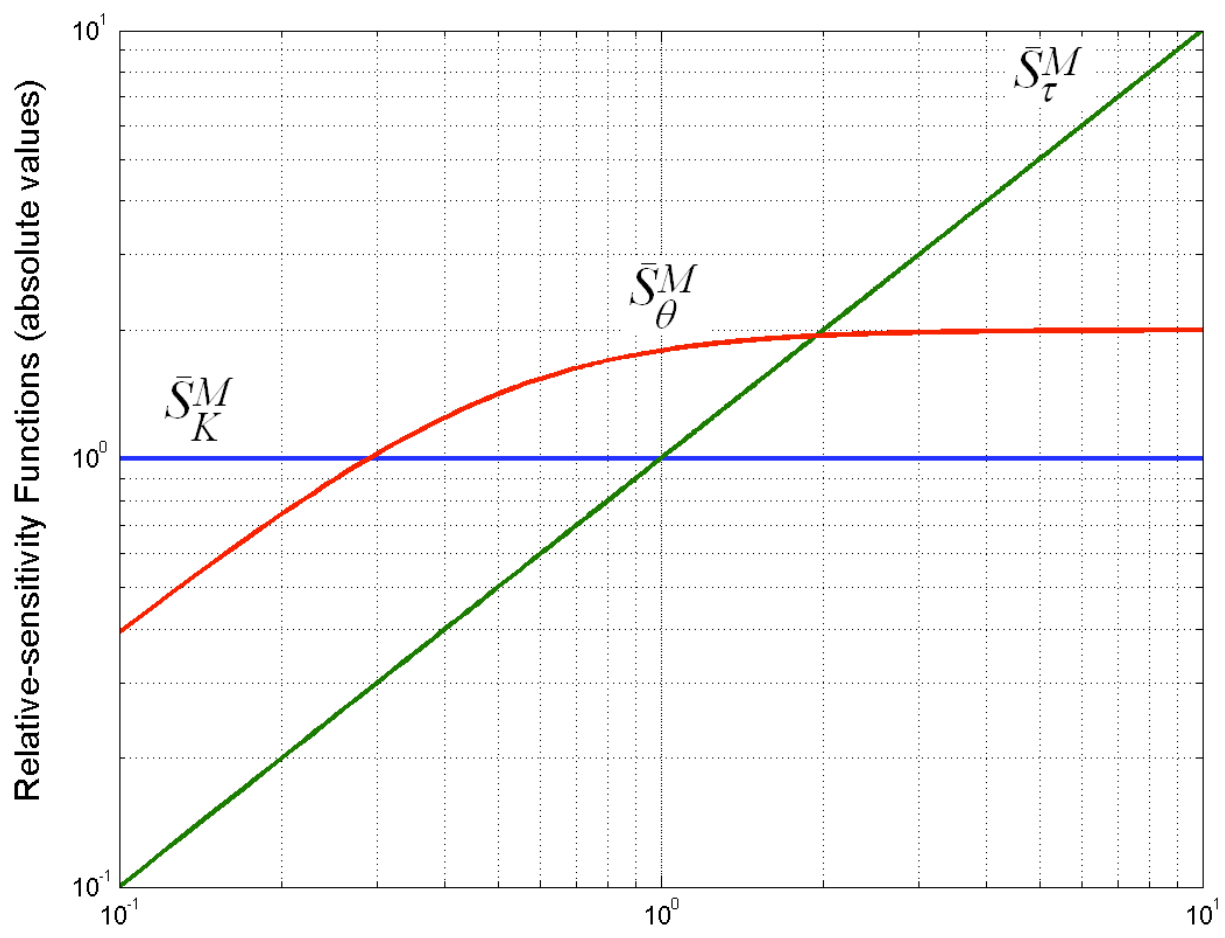

Fig. (1). Relative-sensitivity functions as a function of frequency for the transfer function $M(s)=\frac{Y(s)}{R(s)}=\frac{K e^{-\theta s}}{(\tau s+1)^{2}}$. 


$$
M(s)=\frac{K e^{-\theta s}}{(\tau s+1)^{2}}
$$

Which of the parameters is most important? To answer this question let us compute the relative-sensitivity functions.

$$
\begin{aligned}
& \bar{S}_{K}^{M}=\left.\frac{\partial M}{\partial K}\right|_{\mathrm{NOP}} \frac{K_{0}}{M_{0}}=1 \\
& \bar{S}_{\theta}^{M}=\left.\frac{\partial M}{\partial \theta}\right|_{\mathrm{NOP}} \frac{\theta_{0}}{M_{0}}=-s \theta_{0} \\
& \bar{S}_{\tau}^{M}=\left.\frac{\partial M}{\partial \tau}\right|_{\mathrm{NOP}} \frac{\tau_{0}}{M_{0}}=\frac{-2 s \tau_{0}}{\tau_{0} s+1}
\end{aligned}
$$

The magnitudes of these three relative-sensitivity functions are plotted in Fig. (1) using Stark's [28] normal values of 32, 1 and 2 for $K_{0}, \theta_{0}$ and $\tau_{0}$, respectively. By looking at these plots, we can see that for low frequencies, e.g., $\omega<0.3, K$ has largest magnitude, for mid range frequencies, e.g., $\omega \approx 1, \tau$ has largest magnitude, and for high frequencies, e.g., $\omega>2, \theta$ has largest magnitude.

Now, of course, this was a simple example. And for this example, most people would intuitively say that the gain is the most important parameter for low frequencies (i.e. steady state) and the time-delay is the most important parameter for high frequencies. But it was still nice that this sensitivity analysis gave us a quantitative justification for our intuition.

\subsection{Limitations of the Relative-Sensitivity Function}

The relative-sensitivity function is limited in usefulness in analytic studies for three reasons [14]. First, the relativesensitivity functions are different in the time and in the frequency domains. Second the relative-sensitivity function is the product of two functions (the partial derivative and the original function), and the Laplace transform of a product is not the product of the Laplace transforms, e.g.

$$
\mathrm{L}(x(t) \times y(t)) \neq \mathrm{L}(x(t)) \times \mathrm{L}(y(t))
$$

Therefore, you cannot use Laplace transforms to get time-domain solutions. And finally if the function being analyzed is a time function, then it may take on very small values, which would give erroneous results, or it might even take on values of zero, and division by zero is frowned upon.

\subsection{The Semirelative-Sensitivity Function}

We have used the absolute-sensitivity function to see when a parameter had its greatest effect on the step response of a system, and we have used the relative-sensitivity function to see which parameter had the greatest effect on the transfer function. Now suppose we wish to compare parameters, but we want to look at the step response and not the transfer function. What happens if we try to use the relativesensitivity function to compare parameter effects on the step response? We get into trouble. For a step response, the normal output value $y_{0}$ typically varies from 0 to 1 and division by zero is undefined. Furthermore, the relative-sensitivity function gives undue weight to the beginning of the response when $y_{0}$ is small. Therefore, let us investigate the use of the semirelative-sensitivity function, which is defined as

$\tilde{S}_{\alpha}^{F}=\left.\frac{\partial F}{\partial \alpha}\right|_{\mathrm{NOP}} \alpha_{0}$

where NOP and the subscript 0 mean that all functions and parameters assume their normal operating point values.

As can be seen by the definition, semirelative-sensitivity functions will have the same shape as absolute-sensitivity functions. They are just multiplied by the constant parameter values. But this scaling allows comparisons to be made of the effects of the various parameters. Of course, when a parameter has a value of zero, its semirelative sensitivity function is also zero.

\section{Example 1. The Process Model (Continued)}

Let us now return to the process model, $f(x, y)=z=A x^{2}+B y^{2}+C x y+D x+E y+F . \quad$ Would semirelative-sensitivity functions be better than relativesensitivity functions? There would be little difference, because $z$ is not a function of time and we can prevent it from taking on a zero value. We could get the semirelativesensitivity functions by multiplying the relative-sensitivity functions by $z_{0}$, which is 2 . Thus, all of the first-order relative-sensitivity functions would be multiplied by 2 , which would not change the relative rankings. The second-order relative-sensitivity functions would all be multiplied by 4 , once again, no rankings change. Therefore, for the process model we can use either relative or semirelative-sensitivity functions: it does not make a difference.

\section{Example 2. A Double-Pole System with a Time-Delay (Continued)}

The transfer function is

$M(s)=\frac{K e^{-\theta s}}{(\tau s+1)^{2}}$

The step response of the system is

$Y_{s r}(s)=\frac{K e^{-\theta s}}{s(\tau s+1)^{2}}$

and the semirelative-sensitivity function of the step response with respect to $K$ is

$$
\tilde{S}_{K}^{Y_{s r}}(s)=\frac{e^{-\theta_{0} s}}{s\left(\tau_{0} s+1\right)^{2}} K_{0}
$$

which transforms into

$$
\tilde{S}_{K}^{y_{s r}}(t)=K_{0}\left(1-e^{-\left(t-\theta_{0}\right) / \tau_{0}}-\frac{t-\theta_{0}}{\tau_{0}} e^{-\left(t-\theta_{0}\right) / \tau_{0}}\right) \text { for } t>\theta_{0}
$$


The semirelative-sensitivity function of the step response with respect to $\theta$ is

$$
\tilde{S}_{\theta}^{Y_{s r}}(s)=\frac{-K_{0} e^{-\theta_{0} s}}{\left(\tau_{0} s+1\right)^{2}} \theta_{0}
$$

which transforms into

$$
\tilde{S}_{\theta}^{y_{s r}}(t)=-\frac{K_{0} \theta_{0}}{\tau_{0}^{2}}\left(\left(t-\theta_{0}\right) e^{-\left(t-\theta_{0}\right) / \tau_{0}}\right) \text { for } t>\theta_{0}
$$

The semirelative-sensitivity function of the step response with respect to $\tau$ is

$$
\tilde{S}_{\tau}^{Y_{s r}}(s)=\frac{-2 K_{0} e^{-\theta_{0} s}}{\left(\tau_{0} s+1\right)^{3}} \tau_{0}
$$

which transforms into

$$
\tilde{S}_{\tau}^{y_{s r}}(t)=\frac{-K_{0}}{\tau_{0}^{2}}\left(\left(t-\theta_{0}\right)^{2} e^{-\left(t-\theta_{0}\right) / \tau_{0}}\right) \text { for } t>\theta_{0}
$$

These three semirelative-sensitivity functions are plotted in Fig. (2) using the normal values previous used, namely 32, 1 and 2 for $K_{0}, \theta_{0}$ and $\tau_{0}$, respectively. This sensitivity analysis gives us a wealth of information.

It says that if our model does not match the physical response early in the rise, then we should adjust the time-delay of the model; because in the beginning, the time-delay has its greatest effect, while the sensitivity functions of the gain and the time constant are still small. If we wish to affect the steady state behavior of the model, then we should change the gain, because the effects of the time-delay and the time constant will have decayed to zero. It also tells us that the time constant will have its greatest effect in the middle of the movement. Sometimes a sensitivity analysis like this will show parameters that have their peaks at the same time; these parameters can be treated as a group with trade-offs between their individual values.

Once again, the results of the sensitivity analysis agree with our intuitions: the time-delay has its greatest effect in the beginning of the movement, the time constant has its greatest effect in the middle of the movement, and the gain has its greatest effect at the end of the movement.

\section{Example 3. A Tradeoff Study}

In modeling dynamic systems, most sensitivity functions are functions of time or frequency, but this is not a prerequisite for sensitivity analyses. For example, let us compute the semirelative-sensitivity functions for a tradeoff study. Tradeoff studies provide a rational method for choosing among alternatives when simultaneously considering multiple alternatives with many criteria [31-33]. Tradeoff studies are often called trade studies. In the tradeoff study of Tables $\mathbf{3}$ and $\mathbf{4}$, the alternative ratings are computed with linear ad-

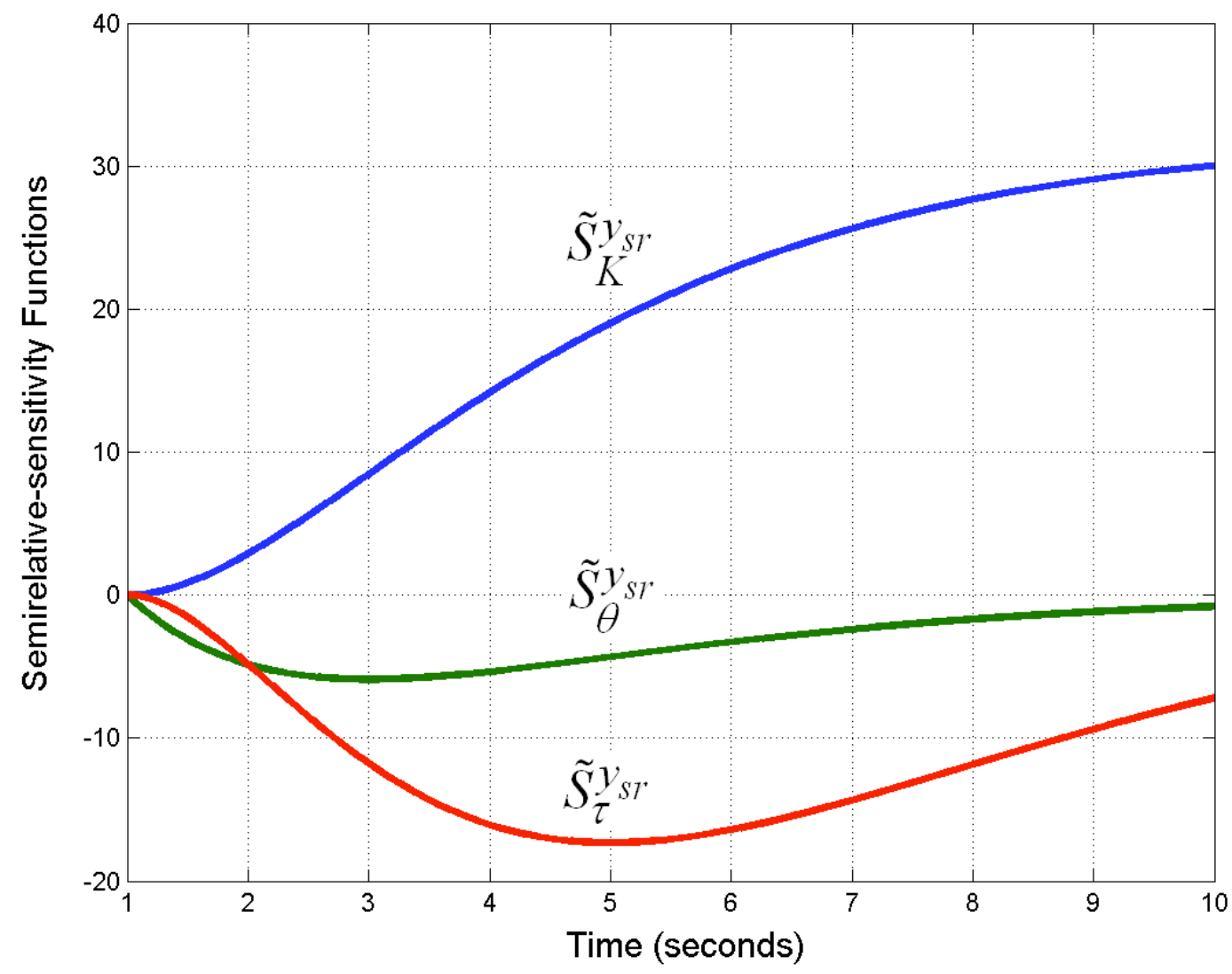

Fig. (2). Semirelative-sensitivity functions of the step response $Y_{s r}(s)=\frac{K e^{-\theta s}}{s(\tau s+1)^{2}}$ with respect to $K, \theta$ and $\tau$. 
Table 3. A Generic Tradeoff Study

\begin{tabular}{|c|c|c|c|}
\hline Criteria & Weight of Importance & Alternative-1 & Alternative-2 \\
\hline \hline Criterion-1 & $W t_{1}$ & $S_{11}$ & $S_{12}$ \\
\hline Criterion-2 & $W t_{2}$ & $S_{21}$ & $S_{22}$ \\
\hline Alternative Rating & & $S u m_{1}=W t_{1} \times S_{11}+W t_{2} \times S_{21}$ & $S u m_{2}=W t_{1} \times S_{12}+W t_{2} \times S_{22}$ \\
\hline
\end{tabular}

Table 4. Tradeoff Study Numerical Example

\begin{tabular}{|c|c|c|c|}
\hline \multirow{2}{*}{ Criteria } & Weight of Importance & Umpire's Assistant & Alternatives \\
\cline { 3 - 4 } & & 0.67 & 0.33 \\
\hline \hline Accuracy of the call & 0.75 & 0.83 & 0.17 \\
\hline Silence of Signaling & 0.25 & 0.71 (The winner) & 0.29 \\
\hline Sum of weight times score & & Seeing Eye Dog \\
\hline
\end{tabular}

dition of the weighted scores. That is, Sum $_{1}=W t_{1} \times S_{11}+W t_{2} \times S_{21}$

and $\operatorname{Sum}_{2}=W t_{1} \times S_{12}+W t_{2} \times S_{22}$.

Table 4 gives numerical values for one particular tradeoff study, The Umpire's Assistant (http://www.sie.arizona.edu/ sysengr/sie577/UmpiresAssistant.doc).

Now we want to perform a sensitivity analysis of this tradeoff study. We want to discover which parameters could change the recommendations of the tradeoff study. Therefore, an appropriate performance index is the rating for the highest rated alternative (alternative-1) minus the rating for second highest rated alternative (alternative-2).

$P I_{1}=\operatorname{Sum}_{1}-$ Sum $_{2}=$

$W t_{1} \times S_{11}+W t_{2} \times S_{21}-W t_{1} \times S_{12}-W t_{2} \times S_{22}=0.420^{\circ}$

We want to compute the semirelative-sensitivity functions for this performance index, with respect to the six parameters. We want to discover which parameter is the most important and plan our future actions.

$\tilde{S}_{W t_{1}}^{P I_{1}}=\left(S_{11}-S_{12}\right) W t_{1}=0.26$

$\tilde{S}_{W t_{2}}^{P I_{1}}=\left(S_{21}-S_{22}\right) W t_{2}=0.16$

$\tilde{S}_{S_{11}}^{P I_{1}}=W t_{1} S_{11}=0.50$
$\tilde{S}_{S_{21}}^{P I_{1}}=W t_{2} S_{21}=0.21$

$\tilde{S}_{S_{12}}^{P I_{1}}=-W t_{1} S_{12}=-0.25$

$\tilde{S}_{S_{22}}^{P I_{1}}=-W t_{2} S_{22}=-0.04$

These sensitivities can be nicely displayed in a table, as in Table 5.

The most important parameter is $S_{11}$. Therefore, we should gather more experimental data and interview more domain experts for this parameter: we should spend extra resources on this parameter. The minus signs for $S_{12}$ and $S_{22}$ merely mean that an increase in either of these parameters will cause a decrease in the performance index.

A problem with this performance index is that if $S_{11}=S_{12}$, then the sensitivity with respect to $W t_{1}$ becomes equal to zero. Numerically this is correct, but intuitively it is wrong. So let us consider a performance index that is the rating for just the winning alternative (in this case alternative-1).

$$
P I_{2}=S u m_{1}=W t_{1} \times S_{11}+W t_{2} \times S_{21}
$$

This performance index is more convenient when there are many alternatives and their relative rankings change frequently. However, a problem with both $P I_{1}$ and $P I_{2}$ is that the sensitivity with respect to $W t_{1}$ does not depend on scores

Table 5. Analytic Semirelative-Sensitivity Function Values for $P I_{1}$, the Difference of the Alternative Ratings

\begin{tabular}{|c|c|c|c|}
\hline \multirow{2}{*}{ Criteria } & Weight of Importance & Alternatives \\
\cline { 3 - 4 } & & Umpire's Assistant & Seeing Eye Dog \\
\hline \hline Accuracy of the call & $\tilde{S}_{W t_{1}}^{P I_{1}}=0.26$ & $\tilde{S}_{S_{11}}^{P I_{1}}=0.50$ & $\tilde{S}_{S_{12}}^{P I_{1}}=-0.25$ \\
\hline Silence of Signaling & $\tilde{S}_{W t_{2}}^{P I_{1}}=0.16$ & $\tilde{S}_{S_{21}}^{P I_{1}}=0.21$ & $\tilde{S}_{S_{22}}^{P I_{1}}=-0.04$ \\
\hline
\end{tabular}


Table 6. Analytic Semirelative-Sensitivity Function Values for $\mathrm{PI}_{3}$, the Sum of All Weight Times Scores

\begin{tabular}{|c|c|c|c|}
\hline \multirow{2}{*}{ Criteria } & Weight of Importance & Umpire's Assistant & Alternatives \\
\cline { 3 - 4 } & $\tilde{S}_{W t_{1}}^{P I_{3}}=0.38$ & $\tilde{S}_{S_{11}}^{P I_{3}}=0.25$ & $\tilde{S}_{S_{12}}^{P I_{3}}=0.12$ \\
\hline \hline Accuracy of the call & $\tilde{S}_{W t_{2}}^{P I_{3}}=0.13$ & $\tilde{S}_{S_{21}}^{P I_{3}}=0.10$ & $\tilde{S}_{S_{22}}^{P I_{3}}=0.02$ \\
\hline Silence of Signaling & & & \\
\hline
\end{tabular}

for the nonwinning alternatives and we do want the sensitivities to depend on the other parameters. The following performance index solves both of these problems.

$$
P I_{3}=\frac{1}{m} \sum_{i=1}^{n} \sum_{j=1}^{m} W t_{i} S_{i j}
$$

where $n$ is the number of criteria and $m$ is the number of alternatives. This performance index is the sum of all the weight times scores in the whole matrix. With this performance index the semirelative-sensitivity functions are

$\tilde{S}_{W t_{i}}^{P I_{3}}=\left.\frac{\partial P I_{3}}{\partial W t_{i}}\right|_{\text {NOP }} W t_{i}=\frac{1}{m} \sum_{j=1}^{m} W t_{i} S_{i j}$ and $\tilde{S}_{S_{i j}}^{P I_{3}}=\frac{W t_{i} S_{i j}}{m}$.

They can be calculated and displayed directly with a spreadsheet as shown in Table $\mathbf{6}$.

Each cell states the sensitivity of the whole matrix with respect to that element. Here are all of the sensitivity functions.

$$
\begin{aligned}
& P I_{3}=\frac{1}{m} \sum_{i=1}^{n} \sum_{j=1}^{m} W t_{i} S_{i j} \\
& \frac{\partial P I_{3}}{\partial W t_{i}} \mid=\frac{1}{m} \sum_{j=1}^{m} S_{i j} \\
& \tilde{S}_{W t_{i}}^{P I_{3}}=\left.\frac{\partial P I_{3}}{\partial W t_{i}}\right|_{\mathrm{NOP}} W t_{i_{0}}=\frac{1}{m} \sum_{j=1}^{m} W t_{i} S_{i j} \\
& \frac{\partial P I_{3}}{\partial S_{i j}}=\frac{W t_{i}}{m} \\
& \tilde{S}_{S_{i j}}^{P I_{3}}=\frac{W t_{i} S_{i j}}{m}
\end{aligned}
$$

$\frac{\partial^{2} P I_{3}}{\partial W t_{i} \partial S_{i j}}=\frac{1}{m}$

$\tilde{S}_{W t_{i}-S_{i j}}^{P I_{3}}=\frac{W t_{i} S_{i j}}{m}$

$\frac{\partial^{2} P I_{3}}{\partial W t_{i}^{2}}=0$

$\tilde{S}_{W t_{i}^{2}}^{P I_{3}}=0$

$\frac{\partial^{2} P I_{3}}{\partial W t_{i} \partial W t_{k}}=0$

$\tilde{S}_{W t_{i}-W t_{k}}^{P I_{3}}=0$

$\frac{\partial^{2} P I_{3}}{\partial S_{i j}^{2}}=0$ This means any combination of $S_{i j}$ 's.

$\tilde{S}_{S_{i j}^{2}}^{P I_{3}}=0$

$\tilde{S}_{S_{i j}}^{P I_{3}}=\tilde{S}_{W t_{i}-S_{i j}}^{P I_{3}}$

Although these equations may look formidable, they are easy to compute with a spreadsheet. For example $\sum_{k=1}^{n} W t_{k} S_{k j}$ is merely the sum of the weight times scores in column $k$ $\left(\operatorname{Sum}_{k}\right)$ and this is already in the spreadsheet. Furthermore, because $\tilde{S}_{S_{i j}}^{P I_{3}}=\tilde{S}_{W_{t}-S_{i j}}^{P I_{3}}$ and the rest of the second order sensitivities are zero, Table 6 is complete: it has all of the sensitivities in it. Additional examples are available at http://www.sie.arizona.edu/sysengr/sie554/ with the Spin Coach documents.

Table 7. Analytic Semirelative-Sensitivity Function Values for the Interactions of $\boldsymbol{P I}_{3}$

\begin{tabular}{|c|c|c|c|}
\hline \multirow{2}{*}{ Criteria } & \multirow{2}{*}{ Weight of Importance } & Alternatives \\
\cline { 3 - 4 } & & Umpire's Assistant & Seeing Eye Dog \\
\hline \hline Accuracy of the call & & $\tilde{S}_{W_{1}-S_{11}}^{P I_{3}}=0.25$ & $\tilde{S}_{W_{1}-S_{12}}^{P I_{1}}=0.12$ \\
\hline Silence of Signaling & & $\tilde{S}_{W t_{2}-S_{21}}^{P I_{3}}=0.10$ & $\tilde{S}_{W t_{2}-S_{22}}^{P I_{3}}=0.02$ \\
\hline
\end{tabular}


A tradeoff study can be hierarchical. There can be any number of criteria and each of them can have any number of subcriteria. Appendix A discusses the sensitivity analysis of a hierarchical tradeoff study.

What about interactions? The non-zero semirelativesensitivity functions for interactions are

$\tilde{S}_{W t_{i}-S_{i j}}^{P I_{3}}=\frac{W t_{i_{0}} S_{i j_{0}}}{m}$

Specifically, the semirelative-sensitivity function for the interaction of $W t_{1}$ and $S_{11}$ is

$\tilde{S}_{W_{1}-S_{11}}^{P I_{3}}=\left.\frac{\partial^{2} P I_{3}}{\partial W t_{1} \partial S_{11}}\right|_{\text {NOP }} W t_{1_{0}} S_{10_{0}}=\frac{W t_{1} S_{11}}{m}=0.25$

This is large enough that we should investigate its effect. If we increase $W t_{1}$ by $10 \%, \quad P I_{3}=0.538$, giving $\Delta P I_{3}=0.038$. If we increase $S_{11}$ by $10 \%, P I_{3}=0.525$, giving $\Delta P I_{3}=0.025$. Now, however, if we increase both $W t_{11}$ and $S_{11}$ by $10 \%$ at the same time, $P I_{3}=0.565$, giving $\Delta P I_{3}=0.065$, which clearly is larger than the sum of 0.038 and 0.025 .

\section{Example 4. Sensitivity Analysis of a Risk Analysis}

Let $P_{j}, S_{j}$ and $R_{j}$ be respectively the probability of occurrence, the severity and the risk, for the $j^{\text {th }}$ failure mode. The risk of failure is typically measured as the probability (or frequency) of occurrence times the severity (or consequences), $R_{j}=P_{j} \times S_{j}$. For the performance index, we can use the sum of the risks, $P I=\sum_{j=1}^{n} R_{j}$. (We would get the same basic result if we let the performance index be the difference between the top two risks.) The semirelativesensitivity of the performance index with respect the probability of the $j^{\text {th }}$ failure mode occurring is $\tilde{S}_{P_{j}}^{P I}=S_{j} \times P_{j_{0}}=R_{j}$ and the semirelative-sensitivity function of the performance index with respect the severity of the $j^{\text {th }}$ failure mode is $\tilde{S}_{S_{j}}^{P I}=R_{j} \times S_{j_{0}}=R_{j}$. The largest sensitivities are always those for the largest risk. This means that we should spend extra time and effort estimating the probability and severity of the highest ranked risk, which seems eminently sensible [34, 35].

\section{EMPIRICAL SENSITIVITY FUNCTIONS}

When analytic equations are not available for systems, sensitivity analyses can be performed on the real-world systems. However, when this would take too much time or too many resources, then the sensitivity analyses can be performed on models of the real-world systems. For many models, deriving sensitivity functions is difficult or impossible, so the sensitivity functions are estimated using computer simulations of the models. Numerical Estimation is a technique that can be used to find system sensitivities. Numerical Estimation can be used on physical systems, models of these systems or computer simulations of these models. Numerical Estimation can be used to estimate any of the previously defined sensitivity functions by properly choosing the parameters to be varied. The estimations are performed by running the system, model or simulation at the normal operating point and then at other operating points. Sometimes the changes in parameter values are small and sometimes they are large, which creates unnatural operating points. One problem with using Numerical Estimation is that the number of runs increases geometrically with the number of factors being studied [36]. Kleijnen [17] reviews the literature on how the simulation community handles this complexity.

\subsection{Numerical Estimation}

The Numerical Estimation method is easy to use and evaluate. For many systems, it is the only alternative, if analytic sensitivity functions cannot be derived. In nondeterministic applications where the model is small, it is probably the best empirical method of evaluating sensitivity of a system to parameter changes.

The primary objective of Numerical Estimation is to estimate the sensitivities numerically. In the technique, a function is evaluated at its normal operating point. Then a parameter is increased or decreased and the function is evaluated at this new point. If we take $\Delta f \approx f^{\prime}\left(x_{0}\right) \Delta x$, with $\Delta x=x-x_{0}$, and if $f^{\prime}$ changes slowly, then $f^{\prime}(\zeta) \approx f^{\prime}\left(x_{0}\right)$, with $\zeta$ being a point between $x$ and $x_{0}$, and $f^{\prime}$ being the derivative at that point. The difference between these two function values is given by

$f(x)-f\left(x_{0}\right)=f^{\prime}(\zeta)\left(x-x_{0}\right)$

Table 8. Numerical Example of Interactions for $P I_{3}$, the Sum of the Alternative Ratings

\begin{tabular}{|c|c|c|c|c|c|}
\hline \multirow{2}{*}{ Sensitivity Function } & Normal Values & Values Increased by 10\% & New $\boldsymbol{P I}_{3}$ Values & $\Delta \boldsymbol{P I}_{3}$ & \multirow{2}{*}{ Total Change in $\boldsymbol{P I}_{3}$} \\
\hline \hline$\tilde{S}_{W_{1}}^{P I_{3}}$ & $W t_{1}=0.75$ & $W t_{1}=0.82$ & 0.538 & 0.038 & \multirow{2}{*}{$\sum \Delta P I_{3}=0.063$} \\
\cline { 1 - 4 }$\tilde{S}_{S_{11} I_{3}}$ & $S_{11}=0.67$ & $S_{11}=0.74$ & 0.525 & 0.025 & \\
\cline { 1 - 3 }$\tilde{S}_{W_{1}-S_{11}}^{P I_{3}}$ & $W t_{1}=0.75 S_{11}=0.67$ & $W t_{1}=0.82 S_{11}=0.74$ & 0.565 & 0.065 & \multirow{2}{*}{0.065} \\
\hline
\end{tabular}


If we want to be more precise, then we should say that

$f(x)-f\left(x_{0}\right)=f^{\prime}\left(x_{0}\right)\left(x-x_{0}\right)+\frac{f^{\prime \prime}(\zeta)}{2 !}\left(x-x_{0}\right)^{2}$.

If $\left(x-x_{0}\right)$ and $f^{\prime \prime}$ are small, then the second term on the right is much smaller than the first one. Therefore, usually the difference in function values divided by the difference in the variable estimates the first derivative, $f^{\prime}(\zeta)=\frac{f(x)-f\left(x_{0}\right)}{\left(x-x_{0}\right)}$. Otherwise $f^{\prime \prime}$ has to be taken into account. In practical applications, the third derivative is seldom a concern.

Assume next that $f$ has two variables, $x$ and $y$, and is twice differentiable.

Then

$f(x, y)-f\left(x_{0}, y_{0}\right)=f_{x}^{\prime}\left(x_{0}, y_{0}\right)\left(x-x_{0}\right)+f_{y}^{\prime}\left(x_{0}, y_{0}\right)\left(y-y_{0}\right)$

$+\frac{1}{2 !}\left\{f_{x x}^{\prime \prime}(\zeta, \eta)\left(x-x_{0}\right)^{2}+2 f_{x y}^{\prime \prime}(\zeta, \eta)\left(x-x_{0}\right)\left(y-y_{0}\right)+f_{y y}^{\prime \prime}(\zeta, \eta)\left(y-y_{0}\right)^{2}\right\}$

where $\zeta$ is between $x$ and $x_{0}$ and $\eta$ is between $y$ and $y_{0}$. If either (1) both $\left(x-x_{0}\right)$ and $\left(y-y_{0}\right)$ are small and the second-order derivatives are not large, or (2) the second-order derivatives are small, then the first two terms dominate. Otherwise, the second-order terms should be taken into consideration when estimating derivatives empirically and then computing sensitivities.

\section{Example 3. A Tradeoff Study (Continued)}

Tradeoff studies are often implemented with spreadsheet tools, such as Excel. It is important to do sensitivity analyses of such systems. Sometimes partial derivatives can be computed for such systems, but more often than not, the sensitivity analyses are estimated empirically. Excel 'what-if scenarios' and Add-Ins such as Palisade's TopRank can help determine the sensitivities. Typically, the analyst changes a parameter (input value, weight, scoring function parameter, etc.) by a fixed percentage, such as $10 \%$, and then looks at the change in the metric of interest. Often this is the rating for the top alternative or the difference between the top two recommended alternatives. Then the sensitivity values are computed. Typically, the relative or the semirelativesensitivity functions are used. The semirelative functions are more robust and they are easier to compute. For example, for a $+10 \%$ parameter change the semirelative-sensitivity function approximation is

$\tilde{S}_{\beta}^{F} \approx \frac{\Delta F}{\Delta \beta} \beta_{0}=\frac{\Delta F}{0.1 \beta_{0}} \beta_{0}=10 \Delta F$
Table 9 shows the tradeoff study example with $S_{11}$ increased from 0.67 to 0.74 . After such changes are made in each of the other five parameters, the empirically derived semirelative sensitivities can be computed and displayed as in Table $\mathbf{1 0 .}$

It is not surprising that these are the same results that were obtained in the analytic semirelative sensitivity section of this paper.

But what about the second-order term in the above "definition" of the single-variable derivative? Namely $\frac{f^{\prime \prime}(\zeta)}{2 !}\left(x-x_{0}\right)^{2}$. If the system in question uses the sum of weighted scores combining function (e.g. $\operatorname{Sum}_{1}=W t_{1} \times S_{11}+W t_{2} \times S_{21}$ and $\left.\operatorname{Sum}_{2}=W t_{1} \times S_{12}+W t_{2} \times S_{22}\right)$, then the second derivatives of a single variable are all zero. So an estimation of the first derivatives that ignores second derivatives will be all right. But this is not true for the product combining function (e. g. $F_{1}=S_{11}^{W W_{1}} \times S_{21}^{W t_{2}}$ and $\left.F_{2}=S_{12}^{W t_{1}} \times S_{22}^{W t_{2}}\right)$ or most other common combining functions. See Daniels, Werner and Bahill [31] for explanations of other combining functions.

Next, let us take another look at the derivatives of a function of two variables.

$f(x, y)-f\left(x_{0}, y_{0}\right)=f_{x}^{\prime}\left(x_{0}, y_{0}\right)\left(x-x_{0}\right)+f_{y}^{\prime}\left(x_{0}, y_{0}\right)\left(y-y_{0}\right)$
$+\frac{1}{2 !}\left\{f_{x x}^{\prime \prime}(\zeta, \eta)\left(x-x_{0}\right)^{2}+2 f_{x y}^{\prime \prime}(\zeta, \eta)\left(x-x_{0}\right)\left(y-y_{0}\right)+f_{y y}^{\prime \prime}(\zeta, \eta)\left(y-y_{0}\right)^{2}\right\}$

As an example, for the case of this tradeoff study, let us examine the second-order terms, those inside the \{\} , for two reasons. First, we want to know if they are large and should be included in determining empirical estimates for the derivatives and sensitivities. Second, for this particular case, we want to estimate the effects of sensitivity interactions.

The analytic second-order semirelative-sensitivity function for the interaction of $W t_{1}$ and $S_{11}$ is

$\tilde{S}_{W t_{1}-S_{11}}^{P I_{3}}=\left.\frac{\partial^{2} P I_{3}}{\partial W t_{1} \partial S_{11}}\right|_{\text {NOP }} W t_{1_{0}} S_{11_{0}}=\frac{W t_{1_{0}} S_{11_{0}}}{m}=0.25$

this is about as large as the largest first-order semirelativesensitivity function.

To estimate values for the second order sensitivities, we might be tempted to use the following simple-minded approximation, here shown for $10 \%$ changes in parameter values.

Table 9. Tradeoff Study with $\mathrm{S}_{11}$ Increased by $10 \%$

\begin{tabular}{|c|c|c|c|}
\hline Criteria & Weight of Importance & Umpire's Assistant & Seeing Eye Dog \\
\hline \hline Accuracy of the call & 0.75 & $\mathbf{0 . 7 4}$ & 0.33 \\
\hline Silence of Signaling & 0.25 & 0.83 & 0.17 \\
\hline Sum of weight times score & & $\mathbf{0 . 7 6}$ & 0.29 \\
\hline
\end{tabular}


Table 10. Numerical Estimates for Semirelative-Sensitivity Function for $P I_{3}$, the Sum of the Alternative Ratings Squared, for a Plus $10 \%$ Parameter Perturbation

\begin{tabular}{|c|c|c|c|}
\hline \multirow{2}{*}{ Criteria } & \multirow{2}{*}{ Weight of Importance } & Alternatives \\
\cline { 3 - 4 } & & Umpire's Assistant & Seeing Eye Dog \\
\hline \hline Accuracy of the call & $\tilde{S}_{W t_{1}}^{P I_{3}}=0.38$ & $\tilde{S}_{S_{11}}^{P I_{3}}=0.25$ & $\tilde{S}_{S_{12}}^{P I_{3}}=0.12$ \\
\hline Silence of Signaling & $\tilde{S}_{W t_{2}}^{P I_{3}}=0.13$ & $\tilde{S}_{S_{21}}^{P I_{3}}=0.10$ & $\tilde{S}_{S_{22}}^{P I_{3}}=0.02$ \\
\hline
\end{tabular}

$\tilde{S}_{\alpha-\beta}^{F} \approx \frac{\Delta F \Delta F}{\Delta \alpha \Delta \beta} \alpha_{0} \beta_{0}=\frac{(\Delta F)^{2}}{0.1 \alpha_{0} 0.1 \beta_{0}} \alpha_{0} \beta_{0}=(10 \Delta F)^{2}$

Letting $\alpha=W t_{1}$ and $\beta=S_{11}$ and using the tradeoff study values in Tables 3 and $\mathbf{4}$, incremented by $10 \%$ where it is appropriate, we get

$\tilde{S}_{W t_{1}-S_{11}}^{P I_{3}} \approx(10 \Delta F)^{2}=0.424$

However, this does not match the analytic value of 0.25 . The lesson here is that perturbation step size $\left(x-x_{0}\right)$ should be small. Five and ten percent step sizes are probably too big for some systems. We might be able resolve this conundrum by using a smaller perturbation size. What if we reduce the perturbation step size to $0.1 \%$ ?

$$
\begin{aligned}
& \tilde{S}_{\alpha-\beta}^{F} \approx \frac{\Delta F \Delta F}{\Delta \alpha \Delta \beta} \alpha_{0} \beta_{0}=\frac{(\Delta F)^{2}}{0.001 \alpha_{0} 0.001 \beta_{0}} \alpha_{0} \beta_{0}=(1000 \Delta F)^{2} \\
& \tilde{S}_{W_{1}-S_{11}}^{P I_{3}} \approx(1000 \Delta F)^{2}=0.393
\end{aligned}
$$

This is closer but still not equal to 0.25 , the number we computed as the analytic semirelative sensitivity. Therefore, we need to improve the mathematical formulation of the estimate of the second-order sensitivity interactions.

In the above simple-minded approximation, we changed both parameters at the same time and then compared the value of the function to the value of the function at its normal operating point. However, this is not the correct estimation for the mixed second-partial derivative. To estimate the second-partial derivatives we should start with

$$
\frac{\partial^{2} f\left(\alpha_{0}, \beta_{0}\right)}{\partial \alpha^{2}} \approx \frac{f_{\alpha}^{\prime}\left(\alpha, \beta_{0}\right)-f_{\alpha}^{\prime}\left(\alpha_{0}, \beta_{0}\right)}{\Delta \alpha} \text { with } \alpha=\alpha_{0}+\Delta \alpha .
$$

and by way of approximation for the first-order derivative we have

$\frac{\partial^{2} f\left(\alpha_{0}, \beta_{0}\right)}{\partial \alpha^{2}} \approx \frac{\frac{f\left(\alpha+\Delta \alpha, \beta_{0}\right)-f\left(\alpha, \beta_{0}\right)}{\Delta \alpha}-\frac{f\left(\alpha, \beta_{0}\right)-f\left(\alpha_{0}, \beta_{0}\right)}{\Delta \alpha}}{\Delta \alpha}$

Simplifying this a little bit we get $\frac{\partial^{2} f\left(\alpha_{0}, \beta_{0}\right)}{\partial \alpha^{2}} \approx \frac{f\left(\alpha+\Delta \alpha, \beta_{0}\right)-2 f\left(\alpha, \beta_{0}\right)+f\left(\alpha_{0}, \beta_{0}\right)}{(\Delta \alpha)^{2}}$

The mixed second-partial derivatives can be obtained similarly.

$\frac{\partial^{2} f\left(\alpha_{0}, \beta_{0}\right)}{\partial \alpha \partial \beta} \approx \frac{f_{\alpha}^{\prime}\left(\alpha_{0}, \beta\right)-f_{\alpha}^{\prime}\left(\alpha_{0}, \beta_{0}\right)}{\Delta \beta}$ with $\beta=\beta_{0}+\Delta \beta$.

Now we can estimate the two first-order derivatives and obtain

$\frac{\partial^{2} f\left(\alpha_{0}, \beta_{0}\right)}{\partial \alpha \partial \beta} \approx \frac{\frac{f(\alpha, \beta)-f\left(\alpha_{0}, \beta\right)}{\Delta \alpha}-\frac{f\left(\alpha, \beta_{0}\right)-f\left(\alpha_{0}, \beta_{0}\right)}{\Delta \alpha}}{\Delta \beta}$

Simplifying this we get

$\frac{\partial^{2} f\left(\alpha_{0}, \beta_{0}\right)}{\partial \alpha \partial \beta} \approx \frac{f(\alpha, \beta)-f\left(\alpha_{0}, \beta\right)-f\left(\alpha, \beta_{0}\right)+f\left(\alpha_{0}, \beta_{0}\right)}{\Delta \alpha \Delta \beta}$

Now we apply this to the tradeoff study of Tables $\mathbf{3}$ and $\mathbf{4}$ with $\mathrm{PI}_{3}$ as shown in Table $\mathbf{1 1}$.

Using the values in Table 11, we get

$$
\frac{\partial^{2} P I_{3}}{\partial W t_{1} \partial S_{11}} \approx \frac{0.50063-0.50038-0.50025+0.50000}{m^{*} 0.00075 * 0.00067}=0.5
$$

where $m$ is defined in $\mathrm{PI}_{3}$ as being the number of alternatives, 2 in this case. Now to get the semirelative-sensitivity function we multiply this second-partial derivative by the normal values $W t_{1_{0}}$ and $S_{11_{0}}$

$\tilde{S}_{W t_{1}-S_{11}}^{P I_{1}}=\left.\frac{\partial^{2} P I_{3}}{\partial W t_{1} \partial S_{11}}\right|_{\text {NOP }} W t_{1_{0}} S_{11_{0}}=0.5 W t_{1_{0}} S_{11_{0}}=0.25$

Now, this is the same result that we derived in the analytic semirelative sensitivity section of this paper.

Although we do not use it in this paper, for completeness, we now give the formula for estimating the second-order derivative for a function of only one variable:

$f^{\prime \prime}(\alpha) \approx \frac{f(\alpha-\Delta \alpha)-2 f(\alpha)+f(\alpha+\Delta \alpha)}{\Delta \alpha^{2}}$

There are two important lessons to be learned from this study. First, for a tradeoff study using the sum combining 
Table 10. Values to be Used in Estimating the Second Derivative

\begin{tabular}{|c|c|c|}
\hline Terms & Parameter Values with a 0.1\% Step Size, that is $\Delta W t_{1}=\mathbf{0 . 0 0 0 7 5}$ and $\Delta S_{11}=\mathbf{0 . 0 0 0 6 7}$ & Function Values \\
\hline \hline$f(\alpha, \beta)$ & $W t_{1}=0.75075$ & 0.50063 \\
\hline$f\left(\alpha_{0}, \beta\right)$ & $S_{11}=0.67067$ & \\
\hline$f\left(\alpha, \beta_{0}\right)$ & $S_{11}=0.67067$ & 0.50025 \\
\hline$f\left(\alpha_{0}, \beta_{0}\right)$ & $W t_{1}=0.75075$ & 0.50038 \\
& $W t_{1}=0.75000$ & 0.50000 \\
\hline
\end{tabular}

function and looking only at first derivatives, anything works. However, for most other sensitivity analyses the perturbation step size should be small: $5 \%$ and $10 \%$ perturbations are not acceptable. Second, it is incorrect to estimate the mixed second partial derivative by changing two parameters at the same time and then comparing the resulting value of the function to the value of the function at its normal operating point: estimating mixed second partial derivatives requires evaluation of four not two numerator terms.

\section{DISCUSSION}

Validation. A sensitivity analysis is powerful technique for validating systems. Validation means ensuring that the system suits the customer's actual needs [37]. If a system (and its model) is very sensitive to parameters over which the customer has no control, then it may be the wrong system for that customer. If the sensitivity analysis reveals the most important parameter and that result is a surprise, then it may be the wrong system. If a system is more sensitive to its parameters than to its inputs, then it may be the wrong system or the wrong operating point. If the sensitivities of the model are different from the sensitivities of the physical system, then it may be the wrong model.

In a set of requirements [38], if you delete a requirement, then your completeness measure (perhaps a traceability matrix) should show a vacuity. When you make a decision [33], you should do a sensitivity analysis and then see if changing the most important decision parameters would change your decision. In a tradeoff study [31, 33], the domain experts should agree that the most important criteria identified by the sensitivity analysis are indeed the most important criteria. In a risk analysis [34, 35], the experts should agree that the risks identified by the sensitivity analysis as being the greatest are indeed the greatest risks. After you prioritize a set of items [39], you should do a sensitivity analysis and discover the most important criteria. Then the values for those criteria should be changed to see if it changes the prioritization. These are all validation issues.

Verification. Sensitivity analyses can also be used to help verify systems. Verification means ensuring that the system complies with its requirements and conforms to its design [37]. In a manmade system or a simulation, unexpected excessive sensitivity to any parameter is a verification mistake. Sen- sitivity to interactions should definitely be flagged and studied: such interactions may be unexpected and undesirable.

The types and methods of sensitivity analysis presented in this paper have ranged from precise mathematical definitions yielding exact sensitivities, to empirical methods for estimating sensitivities. Each has its purpose, advantages and shortcomings.

The precise mathematically defined sensitivity functions yield the maximum information about the sensitivity of a system to its inputs and parameters at the expense of requiring a differentiable set of system equations. In the crayfish model (Example 2), the equations were written in terms of time or frequency. The resulting sensitivities were functions of all other inputs and parameters as well as time or frequency. Relative-sensitivity functions, which involve a transform, are difficult to compute correctly, because multiplication in the time domain requires convolution in the frequency domain. Consequently, of the analytical sensitivity functions, the semirelative-sensitivity functions are the most useful.

In this paper, we have shown the importance of using small parameter perturbations in order to get accurate estimates of the derivatives. However, large step sizes are often used to estimate the robustness using broad range sensitivity analyses [40]. For example, for small step sizes, in our process control model, $f(x, y)=z=A x^{2}+B y^{2}+C x y+D x+E y+F$, the output $z$ is most sensitive (in a relative sense) to changes in $F$. But for a step size of 10 (or more), the output $z$ is much more sensitive (relatively) to changes in $y$.

The sensitivities also depend on the operating point. If the normal operating point is changed from $\left(x_{0}, y_{0}\right)=(1,1)$ to $\left(x_{0}, y_{0}\right)=(10,10)$, then the output $z$ becomes most sensitive (relatively) to the input $y$.

Usually it is desirable for a model to be more sensitive to its inputs than to its system parameters. However, this is not true if the system parameters can be changed easily, for example by just turning a knob: in contrast, it is very true if the system parameters are hard to change, for example if they model the temperature or pressure in a large boiler. At the operating point $\left(x_{0}, y_{0}\right)=(1,1)$, our process control example is not sen- 
sitive the inputs, which means we could twiddle with the inputs forever and not be able to control the output. Therefore, this is not a desirable operating point, if the inputs were clean and easily controlled: however, if the inputs were noisy and hard to control, then this would be an excellent operating point. You would not want your car to be insensitive to changes in the position of your steering wheel. However, you do want your car to be insensitive to the gasoline flow rate when you are filing up your gas tank.

Our process control example

$f(x, y)=z=A x^{2}+B y^{2}+C x y+D x+E y+F$ is insensitive to its inputs near the operating point $(1,1)$. That is the $\partial z / \partial x$ and $\partial z / \partial y$ are equal to zero at the $(1,1)$ operating point and higher order derivatives must be used to evaluate the sensitivities. Moreover, these partial derivatives are near zero only near this operating point. Therefore, we should use this operating point only if the inputs are hard to control. Otherwise, we should use a different operating point.

Once the sensitivities of a system to its inputs and parameters are determined, they can be used to guide future research and design. If the sensitivities of a model are quantitatively similar to the sensitivities of the physical system, then the validity of the model is enhanced. Discrepancies can be used to direct improvements of the model or further testing of the physical system.

The examples in this paper were carefully chosen to be simple, but to succinctly illustrate particular points about sensitivity analyses. At one time, they were models of real-world systems. Then the details and domain knowledge were abstracted out, to leave a polished mathematical epitome. This technique was perfected by Wymore [41].

The sensitivity (a linear function) to a parameter of a linear system is not necessarily linear [14]. This can take the form of interactions with other parameters or non-linear direct effects. In the crayfish model of Example 2, the time-delay had no effect on the amplitude of the step response. So doubling or quadrupling its value had no effect. However, the settling time of the step response did depend on the value of the time-delay, but not in a linear manner. The principle of superposition in linear systems applies to inputs and not parameters. In most cases, the effects of changing two parameters independently are different than changing those same two parameters simultaneously.

In general, we do not say that a model is most sensitive to a certain parameter. Rather we must say that a particular facet of a model is most sensitive to a particular parameter at a particular frequency, point in time and operating point. For example, the crayfish transfer function of Example 2 was most sensitive to the gain $K$ at low frequencies, while the step responses were most sensitive to the time delay, $\theta$, at the beginning of the movement. The chosen facet most likely will be related to the question that the model was formulated to answer. Choosing the facet is the key to the sensitivity analysis.

Now, on a different topic, when doing a Taguchi 3-level Design of Experiments (DoE) [42, 43], you pick a normal value, a high value and a low value. If the high and low are some percentage change, then you are doing a relative sensitivity analysis. If the high and low are plus and minus a unit, then you are doing an absolute sensitivity analysis. Alternatively, the high and low could be realistic design options, in which case it does not correspond to any of our sensitivity functions.

Many commercial tradeoff study tools such as Expert Choice, Logical Decisions, Hiview and Top Rank perform sensitivity analyses: we believe they implement relativesensitivity functions. Palisade's @ Risk uses a sophisticated LHS (Latin Hypercube Sampling) technique [17]. However, it is sometimes difficult to determine what type of sensitivity functions they are computing and therefore it is difficult to understand how to use their results.

Sensitivity analyses are helpful when modeling systems with uncertainty. The sensitivity analysis shows which parameters are important and which parameters are not. This allows the allocation of resources to experiments that will reveal more accurate values for the most important parameters. Sensitivity analyses help us to understand how uncertainties in the inputs and model parameters affect the model and its predictions. Although we have not discussed it in this paper, sensitivity analyses can also be used to study uncertainty in model architecture, assumptions and specifications. Sensitivity analyses are used to increase the confidence in the model and its predictions, by providing an understanding of how the model responds to changes in its inputs, the data used to calibrate it, its architecture or its independent variables.

To show which parameters have the greatest affect on the metric of interest, either relative or semirelative-sensitivity functions may be used. However, if the metric of interest is a function of time, then only semirelative-sensitivity functions should be used.

\section{RESUME}

To help validate a model, compute sensitivities for all parameters and inputs. Most systems should be more sensitive to their inputs than to any of their parameters. To help verify a system, compute the sensitivities. Excessive sensitivity to any parameter is a verification mistake. After you build a model, write a set of requirements or design a system, you should study it to see if it makes sense: one of the best ways to study a model is with a sensitivity analysis.

When estimating first derivatives, the step size should be small and the second derivatives must be computed to ensure valid results. When estimating mixed second-partial derivatives, four numerator terms, not two, must be used. Mixed second-partial derivatives are used to determine if the interaction of parameters is important.

This paper has shown many techniques for performing a sensitivity analysis. They have different purposes and give different results. Therefore, when presenting the results of sensitivity analysis, it is very important to state what type of sensitivity analysis was performed. For many sensitivity analyses, this would simply be a statement that the absolute, relative or semirelative-sensitivity functions were computed either analytically or empirically; and the normal operating point and the step size of the parameter perturbations would be given. 


\section{APPENDIX A. HIERARCHICAL TRADEOFF STUDIES}

A tradeoff study can be hierarchical. There can be any number of criteria and each of them can have any number of subcriteria. Table A suggests the structure.

Table A. The Structure of a Hierarchical Tradeoff Study

\begin{tabular}{|c|c|c|c|c|c|}
\hline Criteria & $\begin{array}{c}\text { Normalized } \\
\text { Criteria Weights }\end{array}$ & Subcriteria & $\begin{array}{c}\text { Normalized Subcriteria } \\
\text { Weights }\end{array}$ & $\begin{array}{c}\text { Scores for } \\
\text { Alternative-1 }\end{array}$ & $\begin{array}{c}\text { Scores for } \\
\text { Alternative-2 }\end{array}$ \\
\hline \multirow{4}{*}{ Performance } & \multirow{4}{*}{$C W^{(1)}$} & Subcriteria-1 & $W t_{1}^{(1)}$ & $S_{11}^{(1)}$ & $S_{12}^{(1)}$ \\
\hline & & Subcriteria-2 & $W t_{2}^{(1)}$ & $S_{21}^{(1)}$ & $S_{22}^{(1)}$ \\
\hline & & Subcriteria-3 & $W t_{3}^{(1)}$ & $S_{31}^{(1)}$ & $S_{32}^{(1)}$ \\
\hline & & Subcriteria-4 & $W t_{4}^{(1)}$ & $S_{41}^{(1)}$ & $S_{42}^{(1)}$ \\
\hline \multirow{2}{*}{ Cost } & \multirow{2}{*}{$C W^{(2)}$} & Subcriteria-1 & $W t_{1}^{(2)}$ & $S_{11}^{(2)}$ & $S_{12}^{(2)}$ \\
\hline & & Subcriteria-2 & $W t_{2}^{(1)}$ & $S_{21}^{(2)}$ & $S_{22}^{(2)}$ \\
\hline \multirow{2}{*}{ Schedule } & \multirow{2}{*}{$C W^{(3)}$} & Subcriteria-1 & $W t_{1}^{(1)}$ & $S_{11}^{(3)}$ & $S_{12}^{(3)}$ \\
\hline & & Subcriteria-2 & $W t_{2}^{(3)}$ & $S_{21}^{(3)}$ & $S_{22}^{(3)}$ \\
\hline \multirow{3}{*}{ Risk } & \multirow{3}{*}{$C W^{(4)}$} & Subcriteria-1 & $W t_{1}^{(4)}$ & $S_{11}^{(4)}$ & $S_{12}^{(4)}$ \\
\hline & & Subcriteria-2 & $W t_{2}^{(4)}$ & $S_{21}^{(4)}$ & $S_{22}^{(4)}$ \\
\hline & & Subcriteria-3 & $W t_{3}^{(4)}$ & $S_{31}^{(4)}$ & $S_{32}^{(4)}$ \\
\hline Alternative Ratings & & & & Sum $_{1}$ & $\mathrm{Sum}_{2}$ \\
\hline
\end{tabular}

Of course, not all tradeoff studies would use the criteria of performance, cost schedule and risk, and many other criteria could also be used. In the following formulae there are $k$ criteria and we index them with $l$. The first criterion will have $\mathrm{n}(1)$ subcriteria. The second criterion will have $\mathrm{n}(2)$ sub criteria and the $k^{\text {th }}$ criterion will have $\mathrm{n}(k)$ subcriteria. Within each criterion $(l=1,2, \ldots, k)$, we index the subcriteria with $i ; i$ will run from 1 to $\mathrm{n}(l)$. There are $m$ alternatives and we index them with $j$. For the criteria weights we use $C W^{(l)}$, the subcriteria weights are $W t_{i}^{(l)}$ and the scores are $S_{i j}^{(l)}$. In this example we combine the data with simple linear addition, $\operatorname{Sum}_{j}=\sum_{l=1}^{k} C W^{(l)} \sum_{i=1}^{n(l)} W t_{i}^{(l)} S_{i j}^{(l)}$.

If the tradeoff study is hierarchical, you could create a new sensitivity weights column as follows: remove the hierarchy from the normalized weights column by multiplying each subcriterion by the normalized weight of its super criterion. The resulting sensitivity weights column will then be used in place of the normalized weights column throughout the matrix. But this fails to give the sensitivities for the criteria weights. Therefore, we recommend using this new performance index, $\mathrm{PI}_{5}$.

$P I_{5}=\frac{1}{m} \sum_{l=1}^{k} C W^{(l)} \sum_{i=1}^{n(l)} \sum_{j=1}^{m} W t_{i}^{(l)} S_{i j}^{(l)}$

The first-order partial derivatives and the semirelative sensitivity functions are

$$
\begin{aligned}
& \frac{\partial P I_{5}}{\partial C W^{(l)}}=\frac{1}{m} \sum_{i=1}^{n(l)} \sum_{j=1}^{m} W t_{i}^{(l)} S_{i j}^{(l)} \\
& \tilde{S}_{C W^{(l)}}^{P I_{5}}=\left.\frac{\partial P I_{5}}{\partial C W^{(l)}}\right|_{\mathrm{NOP}} C W^{(l)}=\frac{1}{m} C W^{(l)} \sum_{i=1}^{n(l)} \sum_{j=1}^{m} W t_{i}^{(l)} S_{i j}^{(l)}
\end{aligned}
$$




$$
\begin{aligned}
& \frac{\partial P I_{5}}{\partial W t_{i}^{(l)}}=\frac{1}{m} C W^{(l)} \sum_{j=1}^{m} S_{i j}^{(l)} \\
& \tilde{S}_{W t_{i}^{(l)}}^{P I_{5}}=\frac{1}{m} C W^{(l)} \sum_{j=1}^{m} W t_{i}^{(l)} S_{i j}^{(l)} \\
& \frac{\partial P I_{5}}{\partial S_{i j}^{(l)}}=\frac{1}{m} C W^{(l)} W t_{i}^{(l)} \\
& \tilde{S}_{S_{i j}^{P I_{5}}}=\frac{1}{m} C W^{(l)} W t_{i}^{(l)} S_{i j}^{(l)}
\end{aligned}
$$

The second-order partial derivatives are

$$
\begin{aligned}
& \frac{\partial^{2} P I_{5}}{\partial\left(C W^{(l)}\right)^{2}}=0 \\
& \frac{\partial^{2} P I_{5}}{\partial\left(W t_{i}^{(l)}\right)^{2}}=0 \\
& \frac{\partial^{2} P I_{5}}{\partial\left(S_{i j}^{(l)}\right)^{2}}=0
\end{aligned}
$$

The mixed second-order partial derivatives and the semirelative sensitivity functions are

$$
\begin{aligned}
& \frac{\partial^{2} P I_{5}}{\partial C W^{(l)} \partial W t_{i}^{(l)}}=\frac{1}{m} \sum_{j=1}^{m} S_{i j}^{(l)} \\
& \tilde{S}_{C W^{(l)}-W t_{i}^{(l)}}^{P I_{5}}=\frac{1}{m} C W^{(l)} \sum_{j=1}^{m} W t_{i}^{(l)} S_{i j}^{(l)} \\
& \frac{\partial^{2} P I_{5}}{\partial C W^{\left(l^{*}\right)} \partial W t_{i}^{(l)}}=0 \text { for } l^{*} \neq l \\
& \frac{\partial^{2} P I_{5}}{\partial C W^{(l)} \partial S_{i j}^{(l)}}=\frac{W t_{i}^{(l)}}{m} \\
& \tilde{S}_{C W^{(l)}-S_{i j}^{(l)}}^{P I_{5}}=\frac{1}{m} C W^{(l)} W t_{i}^{(l)} S_{i j}^{(l)} \\
& \frac{\partial^{2} P I_{5}}{\partial W t_{i}^{(l)} \partial W t_{i^{*}}^{\left(l^{*}\right)}}=0 \text { for all } i^{*} \text { and } l^{*} \\
& \frac{\partial^{2} P I_{5}}{\partial W t_{i}^{(l)} \partial S_{i j}^{(l)}}=\frac{C W^{(l)}}{m} \\
& \tilde{S}_{W t_{i}^{(l)}-S_{i j}^{(l)}}^{P I^{(l)}}=\frac{1}{m} C W^{(l)} W t_{i}^{(l)} S_{i j}^{(l)} \\
& \frac{\partial^{2} P I_{5}}{\partial W t_{i}^{(l)} \partial S_{i^{*} j}^{\left(l^{*}\right)}}=0 \text { for } i^{*} \neq i \text { or } l^{*} \neq l
\end{aligned}
$$

Note

$$
\begin{aligned}
& \tilde{S}_{W t_{i}^{(l)}-S_{i j}^{(l)}}^{I_{5}}=\tilde{S}_{C W^{(l)}-S_{i j}^{(l)}}^{P I_{5}}=\tilde{S}_{S_{i j}}^{P I_{5}} \\
& \tilde{S}_{C W^{(l)}-W t_{i}^{(l)}}^{P I_{5}}=\tilde{S}_{W t_{i}^{(l)}}^{P I_{5}}
\end{aligned}
$$

In general the criteria weights are the most important parameters with the subcriteria weights being the next most important. The interaction terms come next and the individual input scores are the least important. Of course, the most important subcriterion weight is usually more important than the least important criterion weight, etc. These generalizations are in concert with our collective intuition about tradeoff studies. 


\section{BIOGRAPHIES}

Eric Smith earned a B.S. in Physics in 1994, an M.S. in Systems Engineering in 2003 and his Ph.D. in Systems and Industrial Engineering in 2006 from the University of Arizona, Tucson. He is currently a Visiting Assistant Professor in the Department of Engineering Management and Systems Engineering, at the Missouri Institute of Science and Technology at Rolla, 65409.

Ferenc Szidarovszky is a professor of Systems and Industrial Engineering at the University of Arizona in Tucson. He was born in Budapest, Hungary and received his B.S., M.S. and Ph.D. in numerical techniques from the Eötvös University of Sciences in Budapest. He received a second Ph.D. in economics from the Budapest University of Economic Sciences in 1977. He was an assistant and an associate professor in the Department of Numerical Analysis and Computer Sciences of the Eötvös University of Sciences. He served as the Acting Head of the Department of Mathematics and Computer Sciences of the University of Horticulture and Food Industry. He was a professor with the Institute of Mathematics and Computer Sciences of the Budapest University of Economic Sciences.

William J. Karnavas was born in Pittsburgh, PA. He earned his B.S. from Carnegie Mellon University in 1984 and his M.S. from the University of Pittsburgh in 1986, both in electrical engineering. He then went to the University of Arizona and received his Ph.D. in Systems and Industrial Engineering in 1992. He currently works for IBM BPTS WebSphere Competency Center, IBM Application \& Integration Middleware Division, Pittsburgh PA. 1Bill@us.ibm.com

A. Terry Bahill is a Professor of Systems Engineering at the University of Arizona in Tucson. He received his Ph.D. in electrical engineering and computer science from the University of California, Berkeley, in 1975. His research interests are in the fields of system design, systems engineering, modeling physiological systems, eye-hand-head coordination, human decision making and systems engineering theory. He has tried to make the public appreciate engineering research by applying his scientific findings to the sport of baseball. Bahill has worked with BAE Systems in San Diego, Hughes Missile Systems in Tucson, Sandia Laboratories in Albuquerque, Lockheed Martin in Eagan MN, Boeing in Kent WA, Idaho National Engineering and Environmental Laboratory in Idaho Falls and Raytheon Missile Systems in Tucson. For these companies he presented seminars on Systems Engineering, worked on system development teams and helped them describe their Systems Engineering Process. He holds a U.S. patent for the Bat Chooser, a system that computes the Ideal Bat Weight for individual baseball and softball batters. He received the Sandia National Laboratories Gold President's Quality Award. He is a Fellow of the Institute of Electrical and Electronics Engineers (IEEE), of Raytheon and of the International Council on Systems Engineering (INCOSE). He is the Founding Chair Emeritus of the INCOSE Fellows Selection Committee. His picture is in the Baseball Hall of Fame's exhibition "Baseball as Amer- ica." You can view this picture at http://www.sie.arizona. edu/sysengr/

\section{ACKNOWLEDGEMENT}

We thank the three anonymous referees for their helpful comments. This work was supported by the Air Force Office of Scientific Research under AFOSR/MURI F49620-03-10377 .

\section{REFERENCES}

[1] C. Stern and E. R. Sherwoods, The origins of genetics: A Mendel source book, Freeman, San Francisco, CA, pp. 308-312, 1966.

[2] J. F. Box, "Gosset, Fisher, and the t distribution," Am. Stat., vol. 35, no. 2, pp. 61-66, summer 1981.

[3] H. W. Bode, Network analysis and feedback amplifier design, Van Nostrand, New York, 1945.

[4] A. Ford and P. C. Gardiner, "A new measure of sensitivity for social system simulation models," IEEE Trans. Syst. Man Cybern., SMC vol. 9, no. 3, pp. 105-114, March 1979.

[5] P. M. Frank, Introduction to system sensitivity theory, Academic, New York, 1978.

[6] F. K. Hsu, A. T. Bahill and L. Stark, "Parametric sensitivity of a homeomorphic model for saccadic and vergence eye movements," Comput. Programs Biomed., vol. 6, no. 2, pp. 108-116, 1976.

[7] A. T. Bahill, J. R. Latimer and B. T. Troost, "Sensitivity analysis of linear homeomorphic model for human movement," IEEE Trans. Syst. Man Cybern., SMC vol. 10, no. 12, pp. 924-929, December 1980.

[8] S. Lehman and L. Stark, "Simulation of linear and nonlinear eye movement models: Sensitivity analyses and enumeration studies of time optimal control," J. Cybern. Inform. Sci., 4, pp. 21-43, 1979.

[9] A. T. Bahill, "Physiological models, Development," in Wiley Encyclopedia of Electrical and Electronics Engineering, J. Webster, Ed. John Wiley \& Sons, vol. 16, pp.427-446, 1999, Second edition, 2007 [Online] Available: http://www.mrw.interscience.wiley.com/ emrw/9780471346081/eeee/article/W1422/current/html\#W1422sec1-0003 [Accessed January 20, 2008].

[10] S. N. Ghadiali, J. Banks and J. D. Swarts, "Finite element analysis of active Eustachian tube function," J. Appl. Physiol., vol. 97, no. 2, pp. 648-654, 2004.

[11] S. J. Yakowitz and F. Szidarovszky, An introduction to numerical computations, Macmillan, New York, 1989.

[12] B. G. Buchanan and E. H. Shortliffe, Rule-based expert systems, Addison-Wesley, Reading, MA, 1984.

[13] A. T. Bahill, Verifying and validating personal computer-based expert systems, Prentice-Hall, Englewood Cliffs, NJ, 1991.

[14] W. J. Karnavas, P. J. Sanchez and A. T. Bahill, "Sensitivity analyses of continuous and discrete systems in the time and frequency domains," IEEE Trans. Syst. Man Cybern., SMC vol. 23, no. 2, pp. 488-501, March-April 1993.

[15] A. Law and D. Kelton, Simulation, modeling and analysis, McGraw Hill, 2000.

[16] J. P. C. Kleijnen, Statistical techniques in simulation, Dekker, New York, 1975.

[17] J. P. C. Kleijnen, "An overview of the design and analysis of simulation experiments for sensitivity analysis," Eur. J. Oper. Res., vol. 164, no. 2, pp. 287-300, July 2005.

[18] P. J. Sanchez and L. W. Schruben, "Simulation factor screening using frequency domain methods: An illustrative example," 18th Winter Simulation Conference, 1986, pp. 366-369.

[19] L. W. Schruben and V. J. Cogliano, "An experimental procedure for simulation response surface model identification," Communications $A C M$, vol. 30, no. 8, pp. 716-730, August 1987.

[20] D. J. Morrice and L. W. Schruben, "Simulation sensitivity analysis using frequency domain experiments," Proceedings 1989 Winter Simulation Conference, 367-373, 1989.

[21] R. Y. Rubinstein and F. Szidarovszky, "Convergence of perturbation analysis estimates for discontinuous sample functions: A general approach," Advances in Applied Probability, vol. 20, no. 1, pp. 59-78, January 1988.

[22] Y. C. Ho and S. Li, "Extensions of infinitesimal perturbation analysis," IEEE Trans. Auto. Cont., vol. 33, no. 5, pp. 427-438, May 1988. 
[23] P. V. Kokotovic and H. K. Khalil, Singular perturbations in systems and control, IEEE Press, New York, 1986.

[24] A. T. Bahill, "A simple adaptive Smith-predictor for controlling time-delay systems," IEEE Cont. Sys. Mag., pp. 16-22, May 1983.

[25] I. D. Landau, Adaptive control: The model reference approach, Dekker, New York, 1979

[26] V. Chatal, Linear programming, Freeman, New York, 1983.

[27] J. E. Oakley and A. O'Hagan, "Probabilistic sensitivity analysis of complex models: a Bayesian approach," J. Royal Stat. Society B, vol. 66, no. 3, pp. 751-769, 2004

[28] L. Stark, Neurological Control Systems, Studies in Bioengineering, Plenum Press, New York, pp. 308-312, 1968.

[29] A. Saltelli, S. Tarantola, F. Campolongo and M. Ratto, Sensitivity analysis in practice: A guide to assessing scientific models, Wiley, Hoboken, NJ, 2004.

[30] W. Nicholson, Microeconomic theory: Basic principles and extensions, The Dryden Press, Hinsdale, IL, 1978.

[31] J. Daniels, P. W. Werner and A. T. Bahill, "Quantitative methods for tradeoff analyses," Syst. Eng., vol. 4, no. 3, pp. 190-212, fall 2001.

[32] E. D. Smith, M. Piattelli-Palmarini and A. T. Bahill, "Cognitive biases affect the acceptance of tradeoff studies," in Decision Modeling and Behavior in Uncertain and Complex Environments, $\mathrm{T}$. Kugler, J. C. Smith, T. Connolly and Y. J. Son., Eds. Springer, Science + Business Media, Cambridge, MA, in press 2008.

[33] E. D. Smith, Y. J. Son, M. Piattelli-Palmarini and A. T. Bahill, "Ameliorating the effects of cognitive biases on tradeoff studies," Syst. Eng., vol. 10, no. 3, pp. 222-240, fall 2007
[34] E. D. Smith and A. T. Bahill, "Risk Analysis," Proceedings of $17^{\text {th }}$ Annual International Symposium of INCOSE, San Diego, CA, June 24-28, 2007

[35] A. T. Bahill and E. D. Smith, "An industry standard risk analysis technique," Eng. Manag. J., 2008.

[36] D. G. Cacuci, Sensitivity and uncertainty analysis, Chapman \& Hall / CRC Press, Boca Raton, FL, 2005.

[37] A. T. Bahill and S. J. Henderson, "Requirements development, verification and validation exhibited in famous failures," Syst. Eng., vol. 8 , no. 1, pp. 1-14, spring 2005.

[38] A. T. Bahill, and F. F. Dean, "Discovering system requirements," in Handbook of Systems Engineering and Management, A. P. Sage, and W. B. Rouse, Eds. John Wiley \& Sons, pp. 175-220, 1999, second edition in press 2008.

[39] R. Botta and A. T. Bahill, "A prioritization process," Eng. Manag. $J$., vol. 19, no. 4, pp. 20-27, December 2007.

[40] W. W. Wakeland, R. H. Martin and D. Raffo, "Using design of experiments, sensitivity analysis, and hybrid simulation to evaluate changes to a software development process: a case study," Software Process: Improvement and Practice, vol. 9, no. 2, pp. 107-119, 2004.

[41] A. W. Wymore, Model-based systems engineering, CRC Press Inc., Boca Raton, FL, 1993

[42] J. J. Pignatiello and J. S. Ramberg, "Off-Line Quality Control, Parameter Design, and the Taguchi Method, a Discussion." J. Qual. Tech., vol. 17, no. 4, pp. 198-206, October 1985.

[43] J. S. Hunter, "Statistical design applied to product design," J. Qual. Tech., vol. 17, no. 4, pp. 210-221, October 1985. 